\title{
VMCP-VBAP Regimen
}

National Cancer Institute

\section{Source}

National Cancer Institute. VMCP-VBAP Regimen. NCI Thesaurus. Code C64839.

A regimen consisting of vincristine, melphalan, cyclophosphamide and prednisone

(VMCP) alternating with vincristine, carmustine (BCNU), doxorubicin and prednisone (VBAP), used for the treatment of plasma cell myeloma. 